\title{
Tract-Based Spatial Statistics in Preterm-Born Neonates Predicts Cognitive and Motor Outcomes at 18 Months
}

\author{
E.G. Duerden, J. Foong, V. Chau, H. Branson, K.J. Poskitt, R.E. Grunau, A. Synnes, J.G. Zwicker, and S.P. Miller
}

\begin{abstract}
BACKGROUND AND PURPOSE: Adverse neurodevelopmental outcome is common in children born preterm. Early sensitive predictors of neurodevelopmental outcome such as MR imaging are needed. Tract-based spatial statistics, a diffusion MR imaging analysis method, performed at term-equivalent age (40 weeks) is a promising predictor of neurodevelopmental outcomes in children born very preterm. We sought to determine the association of tract-based spatial statistics findings before term-equivalent age with neurodevelopmental outcome at 18-months corrected age.
\end{abstract}

MATERIALS AND METHODS: Of 180 neonates (born at 24-32-weeks' gestation) enrolled, 153 had DTI acquired early at 32 weeks' postmenstrual age and 105 had DTI acquired later at 39.6 weeks' postmenstrual age. Voxelwise statistics were calculated by performing tract-based spatial statistics on DTI that was aligned to age-appropriate templates. At 18-month corrected age, 166 neonates underwent neurodevelopmental assessment by using the Bayley Scales of Infant Development, 3rd ed, and the Peabody Developmental Motor Scales, 2nd ed.

RESULTS: Tract-based spatial statistics analysis applied to early-acquired scans (postmenstrual age of 30-33 weeks) indicated a limited significant positive association between motor skills and axial diffusivity and radial diffusivity values in the corpus callosum, internal and external/extreme capsules, and midbrain ( $P<.05$, corrected). In contrast, for term scans (postmenstrual age of 37-41 weeks), tract-based spatial statistics analysis showed a significant relationship between both motor and cognitive scores with fractional anisotropy in the corpus callosum and corticospinal tracts $(P<.05$, corrected). Tract-based spatial statistics in a limited subset of neonates $(n=22)$ scanned at $<30$ weeks did not significantly predict neurodevelopmental outcomes.

CONCLUSIONS: The strength of the association between fractional anisotropy values and neurodevelopmental outcome scores increased from early-to-late-acquired scans in preterm-born neonates, consistent with brain dysmaturation in this population.

ABBREVIATIONS: $A D=$ axial diffusivity; Bayley-III = Bayley Scales of Infant Development, 3rd ed; FA = fractional anisotropy; IVH = intraventricular hemorrhage; $\mathrm{IQR}=$ interquartile range; PDMS-2 = Peabody Developmental Motor Scales, 2nd ed; PMA = postmenstrual age; RD = radial diffusivity; TBSS = tract-based spatial statistics

$\mathbf{T}$ he incidence of very preterm birth (24-32 weeks' gestation) is increasing worldwide, ${ }^{1-4}$ yet surviving neonates still have high

\footnotetext{
Received November 28, 2014; accepted December 23.

From the Department of Paediatrics (E.G.D., J.F., V.C., H.B., S.P.M.), Hospital for Sick Children and University of Toronto, Toronto, Ontario, Canada; Department of Pediatrics (V.C., K.J.P., R.E.G., A.S., J.G.Z., S.P.M.), University of British Columbia, BC Children's and Women's Hospitals, Child and Family Research Institute, Vancouver, British Columbia, Canada; and Department of Occupational Science and Occupational Therapy (J.G.Z.), University of British Columbia, Vancouver, British Columbia, Canada.

This work was supported by the Canadian Institutes of Health Research (MOP79262 to S.P.M. and MOP86489 to R.E.G.) and the NeuroDevNet National Centres of Excellence. S.P.M. is currently Bloorview Children's Hospital Chair in Paediatric Neuroscience and was supported by a Tier 2 Canadian Research Chair in Neonatal Neuroscience and the Michael Smith Foundation for Health Research Scholar Award. R.E.G. is supported by a Senior Scientist award from the Child and Family Research Institute. J.G.Z. is funded by a Michael Smith Foundation for Health Research Scholar Award and the Canadian Child Health Clinician Scientist Program.
}

rates of adverse neurodevelopmental outcomes. ${ }^{5-7}$ While childhood impairment in very preterm born neonates is related to a number of factors, evidence suggests that the severity of white matter injury in the neonatal period is predictive of neurodevelopmental impairment. Examination of white matter development in preterm neonates by using DTI, sensitive to microstructural organization, is increasingly recognized as a promising tool to identify neonates at high risk of neurodevelopmental impairment. ${ }^{8}$

\footnotetext{
Paper previously presented in part at: Annual Meeting of the Pediatric Academic Societies, May 3-6, 2014; Vancouver, British Columbia, Canada.

Please address correspondence to Steven P. Miller, MDCM, MAS, FRCPC, Division of Neurology, Hospital for Sick Children, 555 University Ave, Toronto, ON, Canada M5G, 1X8; e-mail: steven.miller@sickkids.ca

-- Indicates open access to non-subscribers at www.ajnr.org

Indicates article with supplemental on-line photo.

http://dx.doi.org/10.3174/ajnr.A4312
} 


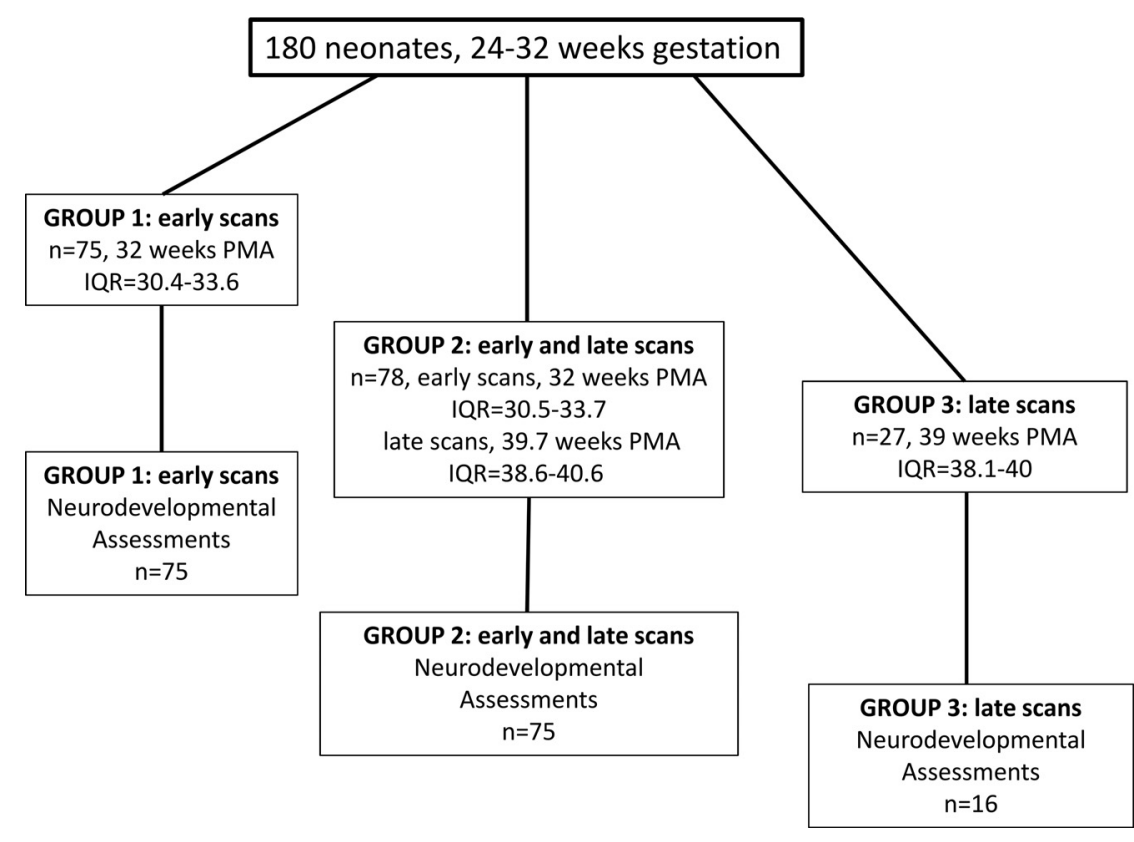

FIG 1. Participant flow chart. Neonate data ( 180 very preterm-born infants of $<32$ weeks' gestation, with 1 or 2 MR imaging scanning sessions including DTI) are separated into 3 groups: group 1, 75 neonates with only 1 early scan near the time of birth (median postmenstrual age at scanning, 32 weeks) (all 75 neonates have neurodevelopmental follow-up data at 18-month corrected age); group 2, 78 neonates with 2 scans, both early (PMA, 32 weeks) and at term-equivalent age (PMA, 39.7 weeks) (75 neonates have follow-up data); group 3, 27 neonates with a late scan (PMA, 39 weeks) (16 neonates have follow-up data).

Previous neonatal brain DTI studies indicated that white matter fractional anisotropy (FA) increases with age, even before myelin is evident on conventional MR imaging sequences. $^{9-11}$ The developmental increase in FA is largely driven by changes in diffusion measures of radial diffusivity (RD) that reflect decreases in membrane permeability of myelinating and premyelinating white matter fiber pathways. ${ }^{11}$ Furthermore, with an ROI approach, FA extracted from DTI scans acquired near birth and term-equivalent age indicated that diffusion parameters correlated with cognitive, language, and motor outcomes. $^{8}$

An alternative to the ROI approach is to analyze the DTI data in a 3D MR image space by using tract-based spatial statistics (TBSS; http://fsl.fmrib.ox.ac.uk/fsl/fslwiki/TBSS), permitting the voxelwise statistical analysis of DTI measures. For example, TBSS performed on scans acquired at term-equivalent age (40 weeks' postmenstrual age [PMA]) in preterm-born neonates detected alterations in white matter microstructure in the absence of overt brain injury. ${ }^{12,13}$ Furthermore, TBSS has been used to examine diffusion measures acquired at termequivalent age and has related them to cognitive and motor outcomes in young preterm-born children. ${ }^{14,15}$ Previous TBSS studies examining outcome measures at 2 years (corrected age) in children born preterm reported that increased FA levels were associated with better outcome. ${ }^{14,15}$ For example, Van Kooij et $\mathrm{al}^{16}$ reported that the increase in $\mathrm{FA}$ in the corpus callosum, fornix, external and internal capsules, superior longitudinal fasciculus, inferior longitudinal and fronto-occipital fasciculi, cingulum, and uncinate fasciculus in their preterm population was positively associated with improved fine motor scores, explained by a decrease in RD. Conversely, the findings of lower FA and increased RD values associated with poorer fine motor scores were interpreted to reflect disruptions in premyelination processes.

Previous TBSS studies have largely focused on scans acquired at termequivalent age in preterm neonates, coinciding with the myelination of major white matter fiber pathways, such as the posterior limb of the internal capsule and the brain stem. ${ }^{13,16}$ However, evidence from histologic and MR imaging studies has indicated that some subcortical structures myelinate at $<30$ weeks of gestation. ${ }^{14,15,17}$ Furthermore, TBSS permits voxelwise statistical analysis of the entire white matter skeleton, a more robust technique compared with an ROI-based approach. Furthermore, DTI analyses performed by using TBSS analysis of DTI measures obtained in neonates have demonstrated that alterations in FA with time were associated with poor outcome. ${ }^{8}$ Therefore, using TBSS applied to early-acquired scans may provide a useful predictive measure of developmental outcome in the first weeks of life. Earlier prediction of neurodevelopmental outcome is highly relevant to clinicians making essential care decisions.

The aim of the present study was to determine whether TBSS analysis of early scans would show a similar association, as seen for term-equivalent-age scans, between white matter microstructure and neurodevelopmental performance assessed at 18 -month corrected age.

\section{MATERIALS AND METHODS Participants}

A total of 180 neonates born very preterm (51\% male, 24-32 weeks' gestation) who were admitted to BC Women's Hospital, Vancouver British Columbia, Canada, were enrolled in the study between April 2006 and September 2010 (Fig 1), as described previously. ${ }^{8}$ The primary inclusion criterion was being born between 24 and 32 weeks of gestation. Neonates with a congenital malformation or syndrome, antenatal infection, or sonographic evidence of a large parenchymal hemorrhagic infarction of $>2 \mathrm{~cm}$ were excluded from the study. The Clinical Research Ethics Board at the University of British Columbia and Children's and Women's Health Centre of British Columbia approved this study, and written informed consent was obtained from the parent or legal caregiver of each infant.

\section{MR Imaging}

Neonates were scanned on an Avanto (Siemens, Erlangen, Germany) 1.5T MR imaging scanner by using VB 13A software. The neonates rested quietly or slept inside a MR-conditional incubator (Lammers Medical Technology, Luebeck, Germany) 
and neonatal head coil (Advanced Imaging Research, Cleveland, Ohio). MR images were obtained as soon the neonate was clinically stable for transport (early scans: median age, 32 weeks; interquartile range [IQR], 30.4-33.7 weeks) and again at term-equivalent age (late scans: median age, 39.6 weeks; IQR, 38.4-40.4 weeks). Neonates underwent anatomic imaging (coronal volumetric T1-weighted images: TR, $36 \mathrm{~ms}$; TE, $9.2 \mathrm{~ms}$; FOV, $200 \mathrm{~mm}$; section thickness, $1 \mathrm{~mm}$; no gap) and axial fast spin-echo T2-weighted imaging (TR, $4610 \mathrm{~ms}$; TE, $107 \mathrm{~ms}$; FOV, $160 \mathrm{~mm}$; section thickness, $4 \mathrm{~mm}$; gap, $0.2 \mathrm{~mm}$ ), followed by a DTI sequence (multirepetition, single-shot echoplanar sequence with 12 gradient directions; TR, $4900 \mathrm{~ms}$; TE, $104 \mathrm{~ms}$; FOV, $160 \mathrm{~mm}$; section thickness, $3 \mathrm{~mm}$; no gap), 3 averages of 2 diffusion weightings of 600 and $700 \mathrm{~s} / \mathrm{mm}^{2}$ (bvalues), and an image without diffusion weighting, resulting in an in-plane resolution of $1.3 \mathrm{~mm}$.

A neuroradiologist (K.J.P.) scored the anatomic images for the severity of white matter injury (none $=0$, minimal $=1$, moderate-severe $=2-3$ combined) and intraventricular hemorrhage $(\mathrm{IVH})($ none $=0$, mild $=1-2$, and moderate-severe $=3-4)$ as reported previously. ${ }^{8}$

\section{Image Analysis}

DTI analyses were performed by using the fMRI of the Brain software library (FSL; http://www.fmrib.ox.ac.uk/fsl/). ${ }^{18}$ Preprocessing included correction for eddy current effects. All diffusion-weighted volumes were linearly registered to 1 nondiffusion-weighted volume for each participant by using affine transformations. ${ }^{19,20}$

The estimated diffusion tensor data were masked to include only the brain by using the Brain Extraction Tool (http://fsl. fmrib.ox.ac.uk/fsl/fslwiki/BET). ${ }^{21}$ A diffusion tensor model was fit to the data at each voxel and to calculate voxelwise fractional anisotropy, mean diffusivity (average of total diffusion within a given voxel), axial diffusivity ( $\mathrm{AD}=$ first eigenvalue: $\lambda 1)$, and radial diffusivity (average of second and third eigenvalues: $\lambda 2$, $\lambda 3)$.

To determine the spatial location of alterations in diffusion measures (FA, mean diffusivity, AD, RD), we processed volumes by using the TBSS pipeline. ${ }^{22}$ FA images were nonlinearly aligned $^{23,24}$ to age-appropriate templates (early preterm scans: $n=24,27-29$ weeks; midpreterm scans: $n=99,30-33$ weeks; late preterm scans: $n=34,34-36$ weeks; term scans: $n=101,37-41$ weeks) to calculate voxelwise statistics.

Data Analysis of TBSS. Voxelwise regression analyses were performed to assess the association of diffusion measures and outcome scores of the Bayley Scales of Infant Development, 3rd ed (Bayley-III) and the Peabody Developmental Motor Scales, 2nd ed (PDMS-2). Cluster-size thresholding was applied to the data, in which the size of the cluster was determined by 500 permutations by using Randomise v.2.9 within the fMRI of the Brain Software Library (http://fsl.fmrib.ox.ac.uk/fsl/fslwiki/ Randomise). A threshold of $P<.05$ (95th percentile of the distribution) was set for the clusters, corrected for multiple comparisons across space. Average FA, AD, and $\mathrm{RD}$ values were then extracted for the whole brain and an ROI in the corpus callosum.

\section{Demographic and Clinical Data Collection}

A neonatal research nurse and neonatal neurologist collected demographic data and clinical variables systematically. Variables of interest included gestational age at birth, cord $\mathrm{pH}$, intensity of resuscitation, days of intubation, any postnatal infections (including positive-culture infection, confirmed necrotizing enterocolitis), patent ductus arteriosus, and chronic lung disease.

\section{Neurodevelopmental Outcomes at 18-Month Corrected Age}

Of the 180 subjects enrolled, 166 children returned for neurodevelopmental follow-up at BC Women's Hospital. Most of the children $(n=155)$ were assessed at 18 - to 21 -month corrected age (median age, 18.7 months; IQR, 18.3-19.5 months), but 11 children were seen between 22 and 37 months. Of the 166 preterm-born children, 75 had early scans, 75 had early and late scans, and 16 were scanned at term-equivalent age. The children's neurodevelopmental abilities were assessed by using the Bayley-III and the PDMS-2 (5 infants were not assessed, total $n=161$ ).

The Bayley-III cognitive, motor, and language composite scores (mean, $100 \pm 15$ ) were calculated. Only the cognitive and language scales were used in the subsequent TBSS analyses. Motor abilities were also assessed by using the PDMS-2, which comprises 6 subtests and generates a Gross Motor and Fine Motor and Total Motor Quotient.

\section{RESULTS}

\section{Clinical Characteristics}

The median gestational age at birth of the 180 neonates included in the study was 27.7 weeks (IQR, 26-29.7 weeks), and the median birth weight was $1022 \mathrm{~g}$ (IQR, 820-1281.3 g). The neonates were grouped on the basis of the number of scans that were included in the final TBSS analyses (group 1, early scans; group 2, early and late scans; group 3, late scans, Fig 1). The details of the clinical characteristics for the groups based on gestational age at MR imaging are listed in Table 1.

\section{Diagnostic MR Imaging Findings}

On the anatomic images, white matter injury was present in up to one-quarter of the most premature babies, but severe IVH was uncommon in this cohort. Diagnostic imaging findings for the groups of neonates stratified by postmenstrual age at the time of the MR imaging are listed in Table 2.

\section{Neurodevelopmental Outcome}

The median age at which the neonates returned for neurodevelopmental follow-up was 18.7-month (IQR, 18.3-19.5 months) corrected age. The median scores on the Bayley-III and on the PDMS- 2 were in the normal range. However, between $6 \%$ and $21 \%$ of neonates scored 1 SD or below $(<84)$ on the Bayley-III or the PDMS-2 (6\%, Bayley-III: cognitive; 21\%, Bayley-III: language; 19\%, PDMS-2: Gross Motor Quotient; 6\%, PDMS-2: Fine Motor Quotient; 16\%, PDMS-2: Total Motor Quotient). The results of the standardized neurodevelopmental assessment scores stratified into groups based on the postmenstrual age at scanning are described in Table 3.

AJNR Am J Neuroradiol 36:1565-71 Aug 2015 www.ajnr.org 
Table 1: Clinical characteristics: separated into groups based on the postmenstrual age at scanning ${ }^{\mathrm{a}}$

\begin{tabular}{|c|c|c|c|c|}
\hline & $\begin{array}{c}\text { 27-29 Weeks } \\
(n=24) \text { (Median) } \\
(\text { IQR) or (No.) (\%) }\end{array}$ & $\begin{array}{c}\text { 30-33 Weeks } \\
(n=99) \text { (Median) } \\
(\text { IQR) or (No.) (\%) }\end{array}$ & $\begin{array}{c}\text { 34-36 Weeks } \\
(n=34) \text { (Median) } \\
(\text { IQR) or (No.) (\%) }\end{array}$ & $\begin{array}{c}\text { 37-41 Weeks } \\
(n=101) \text { (Median) } \\
(I Q R) \text { or (No.) (\%) }\end{array}$ \\
\hline Birth GA (wks) & $27.3(26.1-27.8)$ & $29.3(27.5-30.6)$ & $26.1(25-27.7)$ & $26.9(25.9-29.7)$ \\
\hline Age at MRI (wks) & $29(28.5-29.4)$ & $32(30.9-32.9)$ & $35.1(34.5-36.3)$ & $39.7(38.6-40.6)$ \\
\hline Sex (male) & $12(50 \%)$ & $57(57 \%)$ & $16(47 \%)$ & $54(53 \%)$ \\
\hline Birth weight (g) & 1022.5 (911.5-1171.3) & $1140(957.5-1377.5)$ & $749(607.5-1002.5)$ & $970(805-1270)$ \\
\hline Days of mechanical ventilation & $2(1-5.8)$ & $3(1-10.5)$ & $37.5(20.5-51.3)$ & $11.5(2-51)$ \\
\hline Infection ${ }^{b}$ & $11(46 \%)$ & $31(31 \%)$ & $19(56 \%)$ & $49(49 \%)$ \\
\hline Patent ductus arteriosus & $9(38 \%)$ & $38(38 \%)$ & $27(79 \%)$ & $47(47 \%)$ \\
\hline Chronic lung disease & $5(21 \%)$ & $10(10 \%)$ & $17(50 \%)$ & $27(27 \%)$ \\
\hline
\end{tabular}

Note:-GA indicates gestational age.

${ }^{a}$ One hundred eighty neonates participated. Seventy-five neonates had early scans (near birth), 78 neonates were scanned early and late (birth, term-equivalent age), and 27 neonates were scanned late (term-equivalent age), for a total of 258 scans.

${ }^{\mathrm{b}}$ Infection, culture-positive infection, confirmed necrotizing enterocolitis.

Table 2: Radiologic findings: separated into groups based on the postmenstrual age at scanning ${ }^{\mathrm{a}}$

\begin{tabular}{|c|c|c|c|c|}
\hline & $\begin{array}{c}27-29 \text { Weeks } \\
(n=24) \\
(\text { No. })(\%)\end{array}$ & $\begin{array}{c}\text { 30-33 Weeks } \\
(n=99) \\
(\text { No.) (\%) }\end{array}$ & $\begin{array}{c}\text { 34-36 Weeks } \\
(n=34) \\
(\text { No.) }(\%)\end{array}$ & $\begin{array}{c}37-41 \text { Weeks } \\
(n=101) \\
(\text { No. })(\%)\end{array}$ \\
\hline WMI (moderate/severe) ${ }^{b}$ & $6(25 \%)$ & $15(15 \%)$ & $4(12 \%)$ & $12(12 \%)$ \\
\hline $\mathrm{IVH}(\text { grade } 1 / 2)^{\mathrm{c}}$ & $10(42 \%)$ & $40(40 \%)$ & $18(53 \%)$ & $33(33 \%)$ \\
\hline $\mathrm{IVH}(\text { grade } 3 / 4)^{\mathrm{c}}$ & $1(4 \%)$ & $2(2 \%)$ & $1(3 \%)$ & $2(2 \%)$ \\
\hline Cerebellar hemorrhage & $2(8 \%)$ & $11(11 \%)$ & $5(15 \%)$ & $12(12 \%)$ \\
\hline
\end{tabular}

Note:-WMI indicates white matter injury.

a One hundred eighty neonates participated. Seventy-five neonates had early scans (near birth), 78 neonates were scanned early and late (birth, term-equivalent age), and 27 neonates were scanned late, for a total of 258 scans.

${ }^{b}$ WMI defined as foci exhibiting $T 7$ hyperintensity without $T 2$ hypointensity or by low-intensity $T 7$ foci.

${ }^{c} \mathrm{IVH}$ was graded (none $=0$, mild $=1-2$, and moderate-severe $=3-4$ ) using the Papile system.

Table 3: Neurodevelopmental outcome: separated into groups by postmenstrual age at scanning $^{\mathrm{a}}$

\begin{tabular}{lcccc}
\hline & $\begin{array}{c}\text { 27-29 Weeks } \\
(\boldsymbol{n}=\mathbf{2 2}) \\
(\text { Median) (IQR) }\end{array}$ & $\begin{array}{c}\text { 30-33 Weeks } \\
(\boldsymbol{n}=\mathbf{9 3}) \\
(\text { Median) (IQR) }\end{array}$ & $\begin{array}{c}\text { 34-36 Weeks } \\
(\boldsymbol{n}=\mathbf{3 2}) \\
(\text { Median) (IQR) }\end{array}$ & $\begin{array}{c}\text { 37-41 Weeks } \\
(\boldsymbol{n}=\mathbf{9 4}) \\
(\text { Median) (IQR) }\end{array}$ \\
\hline Age at follow-up (mo) $^{\mathrm{b}}$ & $18.7(18.4-19.2)$ & $18.7(18.3-19.7)$ & $18.6(18.3-19.1)$ & $18.8(18.4-19.4)$ \\
Bayley-III cognitive $^{c}$ & $105(100-110)$ & $110(100-115)$ & $102.5(92.5-110)$ & $105(95-110)$ \\
Bayley-III language $^{c}$ & $101.5(83.5-111.3)$ & $100(91-109)$ & $95.5(83-109)$ & $100(86.8-108.3)$ \\
Bayley-III motor $^{c}$ & $98.5(88.8-105.3)$ & $100(91.8-107)$ & $92.5(84.3-104)$ & $97(88-107)$ \\
PDMS-2 Gross Motor $^{c}$ & $91(87-96)$ & $94(87-98)$ & $89(79-96)$ & $91(87-98)$ \\
PDMS-2 Fine Motor $^{c}$ & $100(97-106)$ & $100(97-103)$ & $97(89.5-103)$ & $100(94-103)$ \\
PDMS-2 Total Motor $^{c}$ & $96(90-101)$ & $96(92-98)$ & $92(84-97)$ & $94(89-98)$
\end{tabular}

a One hundred sixty-six neonates returned for neurodevelopmental follow-up. DTI data were acquired in 75 of the neonates early and in 75 of the neonates at early and late time points (150 scans). Sixteen neonates had late scans for a total of 241 scans

${ }^{\mathrm{b}}$ Age corrected for prematurity.

' The mean composite score in a normative population is $100 \pm 15$.

\section{Diffusion Measures and Neurodevelopmental Outcome}

Cognitive and Language Outcome. The TBSS analysis performed on the diffusion data acquired in neonates scanned at early postmenstrual ages (27-29 weeks, 30-33 weeks, 34-36 weeks) indicated no significant positive or negative association between cognitive and language outcomes and $\mathrm{FA}, \mathrm{AD}$, or $\mathrm{RD}$ values in the white matter skeleton $(P>.05)$.

The TBSS analysis applied to the diffusion data from neonates scanned at 37-41 weeks showed a positive association between FA in a number of white matter tracts and cognitive scores (Fig 2, $P=.02$ ). Specifically, neonates scanned at a postmenstrual age of 37-41 weeks showed a positive relationship between cognitive outcome and FA values in the superior portion of the corona radiata, corticospinal tracts, genu of the corpus callosum, internal capsule, external/extreme capsules, optic radiations, and cerebral peduncle. No voxels demonstrated a negative correlation between FA and cognitive scores. The mean $\mathrm{AD}$ and $\mathrm{RD}$ values were extracted from the regions showing a significant association between FA and cognitive outcome and are plotted in Fig 2. Results indicated that $\mathrm{FA}(R=0.3, P=.03)$ and $\mathrm{RD}(R=-0.2$, $P=.03)$ excluding $\mathrm{AD}(R=-0.1, P=.2)$, were associated with cognitive outcome scores based on Spearman $\rho$ correlations. TBSS applied to the non-FA images indicated a significant negative association with cognitive outcome scores and $\mathrm{RD}$ images $(P=.04)$. No association between cognitive outcome scores and $\mathrm{AD}$ images was found. Results were maintained when removing the data from neonates who had a moderate-severe white matter injury and/or severe IVH (On-line Fig 1). FA values were not significantly associated with language outcomes in neonates scanned at 37-41 weeks $(P=.13)$.

\section{Motor Outcome}

In neonates scanned at early time periods (30-33 weeks), AD and RD images were positively associated with fine motor scores on the PDMS-2, specifically in the territories of the corpus callosum and internal, external/extreme capsules and extending to the cerebral peduncles in the midbrain $(P<.05)$. No association between fine motor scores and FA was evident in neonates scanned at a PMA of 30-33 weeks $(P=.1)$. Additionally, gross and total motor scores were not predicted by diffusion parameters (all $P>.05$ ).

Neonates scanned at later postmenstrual ages (37-41 weeks) also showed a significant positive association of FA with total motor scores in the superior portion of the corona radiata, corticospinal tracts, and the genu of the corpus callosum $(P=.02)$. FA, $\mathrm{AD}$, and RD values were not significantly positively or negatively associated with gross or fine motor scores $(P>.05)$.

In smaller subsets of neonates scanned at other postmenstrual ages (27-29 weeks, 34-36 weeks), FA, AD, and RD were not significantly associated with motor scores $(P>.05)$. 


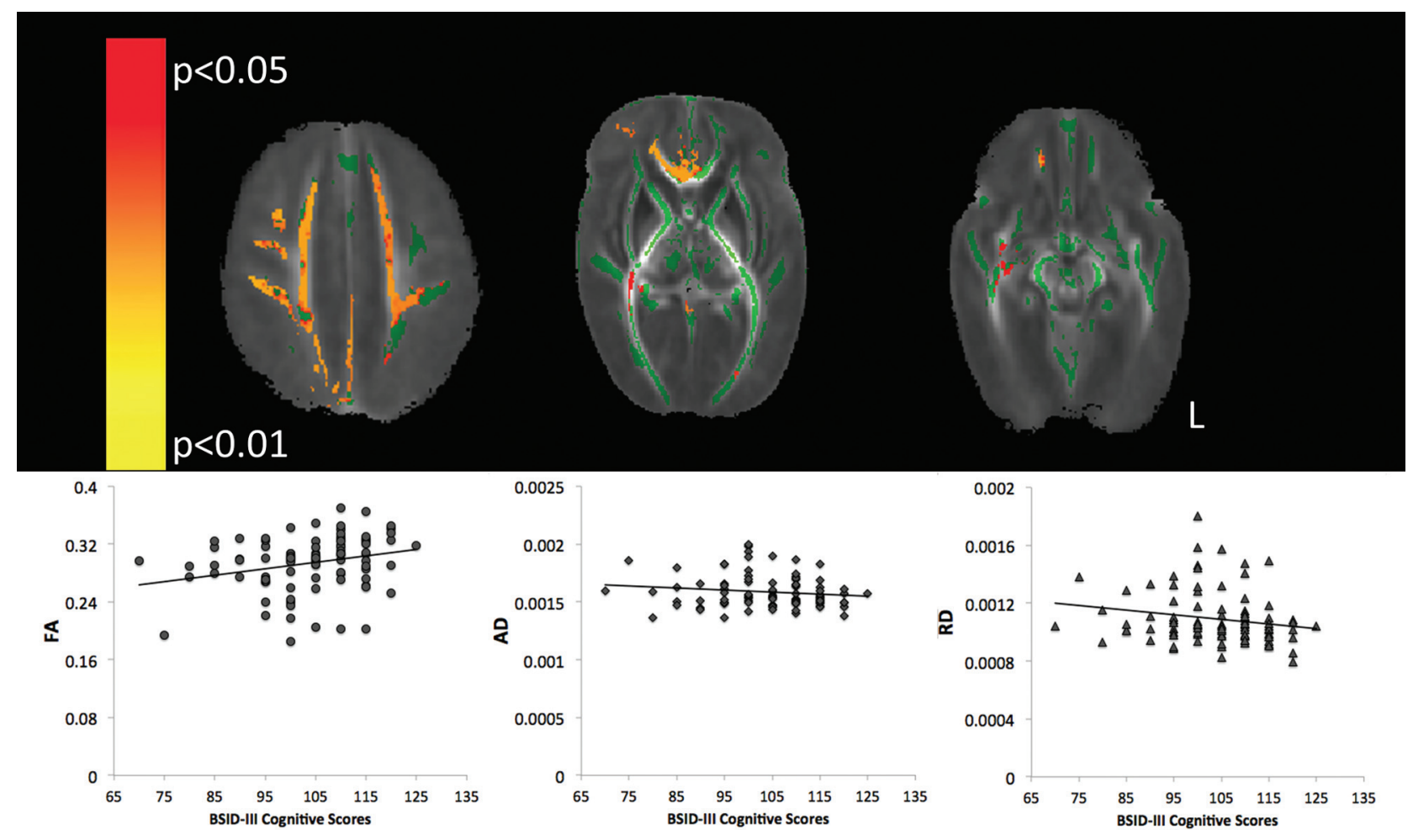

FIG 2. TBSS analysis of term scans (PMA of 37-41-weeks). Top: Mean FA map (red-yellow) demonstrating the significant positive linear association between cognitive scores on the Bayley-III and FA in the territory of the medial prefrontal cortex (left), the genu of the corpus callosum (middle), and portions of the inferior fronto-occipital fasciculus (right, $P<.05$, corrected for multiple comparisons). The mean FA skeleton is shown in green. Bottom: $\mathrm{FA}(R=0.3, P=.03), \mathrm{AD}(R=-0.1, P=.2)$, and $\mathrm{RD}(R=-0.2, P=.03)$ values from the significant clusters in the $\mathrm{FA}$ map. Spearman $\rho$ correlation and an $\alpha$ level are set at .05 .

\section{DISCUSSION}

Using TBSS analysis of diffusion-tensor images, we demonstrated that FA values can be used to predict cognitive outcome at 18 months (corrected age) in preterm-born neonates at term age. Moreover, we demonstrated the utility of using age-specific templates scanned at both early postmenstrual ages (27-29 weeks, 30-33 weeks, 34-36 weeks) and at term age (37-41 weeks) for TBSS analysis in very preterm-born neonates. The analytic method of TBSS offers a number of advantages over hypothesisdirected ROI analyses, in that it describes changes in white matter microstructure in a 3D image space.

TBSS applied to scans acquired around term age, 37-41 weeks' gestation, showed a robust association between FA values in major white matter tracts, with better cognitive and motor performance assessed at 18-month corrected age. The significant findings of higher FA values at 37-41 weeks being correlated with cognitive outcome was largely driven by decreases in RD. Findings are in agreement with a previous TBSS study with pretermborn neonates scanned at term-equivalent age that also reported increased FA in relation to higher cognitive scores and fine motor skills assessed at 2 years. ${ }^{16}$ The biologic significance of increased FA and decreased RD in the absence of changes in $\mathrm{AD}$ is thought to reflect myelination processes leading to reduced permeability. ${ }^{9,25}$ Reductions in RD are associated with the development of oligodendrocyte precursor cells, ${ }^{26}$ while AD increases with the rise in axonal number or increase in axonal caliber. The neonates with relatively lower FA values and higher RD values with poor cognitive outcome may have experienced a disruption in premyelination or myelination processes. ${ }^{27}$

The use of TBSS to predict motor outcome was predictive for neonates scanned as early as 30-33 weeks. Results indicated that fine motor scores were negatively associated with $\mathrm{AD}$ and $\mathrm{RD}$ in white matter fiber pathways located in subcortical regions and the brain stem. Neonates with high $\mathrm{AD}$ and $\mathrm{RD}$ values may be at higher risk for the development of motor abnormalities. Given the widespread changes in $\mathrm{AD}$ and $\mathrm{RD}$ in several white matter fiber pathways, findings may be reflective of the loss of placental growth factors and nutrients due to the early exposure to the extrauterine environment or early systemic illness.

The higher FA values and lower RD values as revealed by using TBSS applied to scans acquired at 30-33 weeks and 37-41 weeks are consistent with myelination patterns seen during typical development. ${ }^{23}$ Myelination does not occur in a uniform process, but rather, different sites myelinate at distinctive times during different time intervals. ${ }^{15}$ Portions of white matter tracts in the forebrain begin to myelinate at $28-29$ weeks. ${ }^{23}$ At $37-40$ weeks of gestation, the posterior limb of the internal capsule and the lateral white matter of the cerebellum are myelinated, while regions of the frontal pole begin to myelinate after 40 weeks. ${ }^{17,18}$ In the current study, FA values in the anterior portion of the corpus callosum extracted from data acquired in neonates scanned at 30-33 weeks were significantly associated with motor outcome. The probabilistic analytic method used by TBSS can detect white matter tracts in areas of low FA, including those regions that are 
sparsely myelinated or unmyelinated. ${ }^{24,28}$ The early-acquired scans (27-29 weeks) analyzed with TBSS did not predict neurodevelopmental outcome, thus indicating a time lag to detect the abnormal white matter maturation that is predictive of cognitive outcome in preterm neonates. The lack of predictive findings from TBSS applied to the early scans is likely reflective of dysmaturation of the white matter, which is an important brain abnormality in preterm neonates. ${ }^{27}$

Language outcomes were not found to be significantly associated with FA values extracted in any of the postmenstrual age groups of interest. Language capabilities in infants of 18-24 months can be strongly influenced by environmental factors. Therefore, the lack of association between linguistic ability and diffusion measures of white matter microstructure seen in the current work may reflect the broad range of language capabilities in children at 18 months of age. An additional consideration is that methodologic limitations of TBSS may have affected the resolution of the arcuate fasciculus, the main white matter fiber pathway subserving speech and language. ${ }^{29}$

\section{CONCLUSIONS}

The development of sensitive MR imaging-based measures of white matter microstructural development in preterm-born neonates is important for the understanding of neurodevelopmental outcome, not only for the early diagnosis and treatment of neonatal brain injury and dysmaturation but also to optimize outcomes for preterm babies. In this study, we have demonstrated that through the development of age-appropriate templates, it was possible to use TBSS to predict motor outcome in scans acquired as early as postmenstrual age of 30-33 weeks. TBSS analysis applied to earlier-acquired scans was not found to be predictive of cognitive outcome. However, cognitive and motor outcomes were predicted by TBSS analysis applied to scans acquired at 37-41 weeks, even when neonates with brain injury were excluded. White matter dysmaturation is increasingly recognized as the primary pathology in contemporary cohorts of preterm neonates. ${ }^{27}$ Thus, the full extent of white matter abnormalities in the preterm neonate may not be apparent on early scans, necessitating follow-up at term-equivalent age.

\section{ACKNOWLEDGMENTS}

The authors thank the families who participated in this research. We also thank Janet Rigney, Sandra Belanger, RN, and Mark Chalmers, RRT (University of British Columbia), for research support, and the staff of the Neonatal Follow-Up Clinic at BC Women's Hospital.

Disclosures: Emma G. Duerden—RELATED: Grant: Canadian Institutes of Health Research, ${ }^{*}$ Comments: This grant provided the funds to conduct the research. Vann Chau—RELATED: Grant: Canadian Institutes of Health Research, ${ }^{*}$ NeuroDevNet National Centres of Excellence; Helen Branson—RELATED: Grant: Canadian Institutes of Health Research, ${ }^{*}$ NeuroDevNet. * Anne Synnes-RELATED: Grant: Canadian Institutes of Health Research grant, ${ }^{*}$ Comments: Canadian Institutes of Health Research grant funded MRI and 18-month patient assessments and research staff; public funding agency/program in Canada;* NeuroDevNet; UNRELATED: Grants/Grants Pending: Canadian Institutes of Health Research grant.* Jill G. Zwicker-RELATED: Grant: Michael Smith Foundation for Health Research Scholar Award,* Canadian Child Health Clinician Scientist Program Career Enhancement Award*; UNRELATED: Grants/Grants Pending: Michael Smith Foundation for Health Research Scholar Award,* Canadian Child Health Clinician Scientist Program Career Enhancement Award*; Travel/Accommodations/Meeting Expenses Unrelated to Activities Listed: used start-up funds and professional development funds from my institution to pay for conference travel/accommodations/registration. Steven P. Miller-RELATED: Grant: Canadian Institutes of Health Research (Principal Investigator) and NeuroDevNet. Ruth E. Grunau-Canadian Institutes of Health Research grant MOP86489 and the NeuroDevNet. *Money paid to the Institution.

\section{REFERENCES}

1. Beck S, Wojdyla D, Say L, et al. The worldwide incidence of preterm birth: a systematic review of maternal mortality and morbidity. Bull World Health Organ 2010;88:31-38

2. Blencowe H, Cousens S, Chou D, et al. Born too soon: the global epidemiology of 15 million preterm births. Reprod Health 2013; 10(suppl 1):S2

3. Chang $\mathrm{HH}$, Larson $\mathrm{J}$, Blencowe $\mathrm{H}$, et al. Preventing preterm births: analysis of trends and potential reductions with interventions in 39 countries with very high human development index. Lancet 2013;381:223-34

4. Blencowe $\mathrm{H}$, Cousens S, Oestergaard MZ, et al. National, regional, and worldwide estimates of preterm birth rates in the year 2010 with time trends since 1990 for selected countries: a systematic analysis and implications. Lancet 2012;379:2162-72

5. D'Onofrio BM, Class QA, Rickert ME, et al. Preterm birth and mortality and morbidity: a population-based quasi-experimental study. JAMA Psychiatry 2013;70:1231-40

6. Herber-Jonat S, Streiftau S, Knauss E, et al. Long-term outcome at age 7-10 years after extreme prematurity: a prospective, two centre cohort study of children born before 25 completed weeks of gestation (1999-2003). J Matern Fetal Neonatal Med 2014;27:1620-26

7. Edwards J, Berube M, Erlandson K, et al. Developmental coordination disorder in school-aged children born very preterm and/or at very low birth weight: a systematic review. J Dev Behav Pediatr 2011;32:678-87

8. Chau V, Synnes A, Grunau RE, et al. Abnormal brain maturation in preterm neonates associated with adverse developmental outcomes. Neurology 2013;81:2082-89

9. Dubois A, Bringuier S, Capdevilla X, et al. Vocal and verbal expression of postoperative pain in preschoolers. Pain Manag Nurs 2008;9:160-65

10. Hüppi PS, Maier SE, Peled S, et al. Microstructural development of human newborn cerebral white matter assessed in vivo by diffusion tensor magnetic resonance imaging. Pediatr Res 1998;44:584-90

11. Partridge SC, Mukherjee P, Henry RG, et al. Diffusion tensor imaging: serial quantitation of white matter tract maturity in premature newborns. Neuroimage 2004;22:1302-14

12. Anjari M, Srinivasan L, Allsop JM, et al. Diffusion tensor imaging with tract-based spatial statistics reveals local white matter abnormalities in preterm infants. Neuroimage 2007;35:1021-27

13. Alexandrou G, Mårtensson G, Skiold B, et al. White matter microstructure is influenced by extremely preterm birth and neonatal respiratory factors. Acta Paediatr 2014;103:48-56

14. Counsell SJ, Maalouf EF, Fletcher AM, et al. MR imaging assessment of myelination in the very preterm brain. AJNR Am J Neuroradiol 2002;23:872-81

15. Kinney HC, Brody BA, Kloman AS, et al. Sequence of central nervous system myelination in human infancy. II. Patterns of myelination in autopsied infants. J Neuropathol Exp Neurol 1988;47:217-34

16. van Kooij BJ, de Vries LS, Ball G, et al. Neonatal tract-based spatial statistics findings and outcome in preterm infants. AJNR Am J Neuroradiol 2012;33:188-94

17. Barkovich AJ. Concepts of myelin and myelination in neuroradiology. AJNR Am J Neuroradiol 2000;21:1099-109

18. Jenkinson M, Beckmann CF, Behrens TE, et al. FSL. Neuroimage 2012;62:782-90

19. Jenkinson M, Smith SM. A global optimisation method for robust affine registration of brain images. Med Image Anal 2001;5:143-56

20. Jenkinson M, Bannister PR, Brady JM, et al. Improved optimisation for the robust and accurate linear registration and motion correction of brain images. Neuroimage 2002;17:825-41 
21. Smith SM. Fast robust automated brain extraction. Hum Brain Mapp 2002;17:143-55

22. Smith SM, Jenkinson M, Johansen-Berg H, et al. Tract-based spatial statistics: voxelwise analysis of multi-subject diffusion data. Neuroimage 2006;31:1487-505

23. Gilles FH, Shankle W, Dooling EC. Myelinated tracts: growth patterns. In: Gilles FH, Leviton A, Dooling EC, eds. The Developing Human Brain: Growth and Epidemiologic Neuropathology. Littleton: John Wright; 1983:118-82

24. Bassi L, Chew A, Merchant N, et al. Diffusion tensor imaging in preterm infants with punctate white matter lesions. Pediatr Res 2011;69:561-66

25. Dubois J, Hertz-Pannier L, Dehaene-Lambertz G, et al. Assessment of the early organization and maturation of infants' cerebral white mat- ter fiber bundles: a feasibility study using quantitative diffusion tensor imaging and tractography. Neuroimage 2006;30:1121-32

26. Drobyshevsky A, Song SK, Gamkrelidze G, et al. Developmental changes in diffusion anisotropy coincide with immature oligodendrocyte progression and maturation of compound action potential. J Neurosci 2005;25:5988-97

27. Back SA, Miller SP. Brain injury in premature neonates: a primary cerebral dysmaturation disorder? Ann Neurol 2014;75: 469-86

28. Bassi L, Ricci D, Volzone A, et al. Probabilistic diffusion tractography of the optic radiations and visual function in preterm infants at term equivalent age. Brain 2008;131:573-82

29. Catani M, Jones DK, ffytche DH. Perisylvian language networks of the human brain. Ann Neurol 2005;57:8-16 